\title{
基于环糊精的超分子手性光化学反应
}

\author{
姚家斌徐梦瑜严志强伍晚花杨 成* \\ (四川大学化学学院 成都 610064)
}

\begin{abstract}
摘要 总结了以环糊精为手性主体分子的超分子手性光化学反应及其特点，内容包括单分子手性光化学反应、双分子 手性光化学反应、手性光催化反应以及手性光反应中的波长效应等四个方面. 着重总结了环糊精对不同光反应底物的 包结特性, 天然和化学修饰的环糊精分子对反应速度、产物的分布和反应选择性的影响以及其作用机理. 探讨了环糊 精作为主体分子用于手性光化学反应所独具的优点, 总结了现阶段研究中存在的问题, 并对该方面工作的发展进行了 展望.
\end{abstract}

关键词 环糊精; 超分子化学; 立体化学, 光化学; 光催化

\section{Supramolecular Photochirogenesis with Cyclodextrins}

\author{
Yao, Jiabin $\quad \mathrm{Xu}$, Mengyu Yan, Zhiqiang Wu, Wanhua Yang, Cheng* \\ (College of Chemistry, Sichuan University, Chengdu 610064)
}

\begin{abstract}
In the present paper, supramolecular chiral photoreactions using cyclodextrins (CDs) as chiral inductors, including monomolecular photochirogenic reactions, bimolecular photochirogenic reactions, photochemical catalysis as well as the wavelength effects in photochirogenesis, have been summarized. This review focused on the binding behavior of CDs with different photosubstrates, the characteristics of chiral photoreactions mediated by CDs, the products distribution and stereoselectivity of chiral photoreactions with CDs and their working mechanisms. This paper concluded the advantages of CDs as host molecules used for chiral photoreactions, the problems existing in these supramolecular photochirogenesis systems and the perspective in this multidisciplinary research field.
\end{abstract}

Keywords cyclodextrins; supramolecular chemistry; stereochemistry; photochemistry; photocatalysis

不对称合成是当代化学的热点研究领域之一，有关 不对称合成的研究在许多方面已经获得了巨大的成功. 然而, 当前有关不对称光化学反应的研究仍然处于不成 熟阶段. 不对称光化学是合成许多具有多环或高张力手 性分子的高效，便捷的方法 ${ }^{[1]}$. 这些具有特殊结构的手 性化合物往往很难通过热化学反应或酶反应等方法来 获得, 因而近年来不对称光化学这一独特领域吸引了众 多化学家的关注. 然而由于有机分子在电子激发态的寿 命很短(往往短于数纳秒), 在如此短的时间尺度内很难 发展出高效的手性诱导; 反应底物在激发态的分子间作 用往往弱于基态, 不利于完成手性源间的手性传递; 此 外处于激发态的有机分子具有非常高的反应活性, 通常 可以不与手性源分子作用而直接发生化学转变. 光化学 反应的这些特点为不对称光反应的手性控制带来了很 大困难, 表现在绝大部分手性光化学反应的对映体过量 值(ee)小于 $50 \%$.

最近的研究工作证实利用超分子相互作用来控制 不对称光化学反应的立体选择性是一个极有前途的方 法 $^{[2 \sim 5]}$. 手性主体分子可以通过包结、络合等作用和光 反应底物形成主客体复合物, 主体分子提供的强非共价 作用和对客体分子的空间约束, 有利于实现高效的手性 传递. 另外，主体分子可以和基态光反应底物形成复合 物，受到光激发后，这种在基态的超分子作用可以传递 到底物的激发状态，从而可以消除激发状态寿命短的不 利影响. 手性修饰的沸石 ${ }^{[6]}$ 、环糊精 $(\mathrm{CD})^{[7]}$ 、手性模 板 ${ }^{[8,9]}$ 、手性液晶 ${ }^{[10]}$ 以及凝胶 ${ }^{[111]}$ 等多种超分子体系已经 被应用于超分子手性光反应的手性诱导体中. 其中, CD 是最先也是被研究得最为广泛的主体分子. 由于在第一 面和第二面上存在有大量的羟基, CD 具有较好的水溶 性. CD 拥有截顶圆雉状空腔体结构 ${ }^{[11 b]}$, 这些空腔能够

* E-mail: yangchengyc@scu.edu.cn

Received August 29, 2013; revised October 14, 2013; published online October 31, 2013.

Project supported by the National Natural Science Foundation of China (Nos. 21372165, 23321061).

国家自然科学基金(Nos. 21372165, 23321061)资助项目. 
通过疏水作用容纳不同大小的客体分子. 目前, 由 6,7 , 8 个葡萄糖单元组成的 $\alpha-, \beta-, \gamma-\mathrm{CD}$ 及许多它们的衍生物 可通过市场获得 ${ }^{[12]}$. 出于这些原因, $\mathrm{CD}$ 在超分子化学 领域扮演了重要角色. 以下几个优势使得 $\mathrm{CD}$ 主体广泛 应用于手性光化学研究: $\mathrm{CD}$ 对波长大于 $220 \mathrm{~nm}$ 的光基 本没有吸收, 因此可以用于大部分光化学反应; $\mathrm{CD}$ 本身 是手性的, 可作为天然的手性源; 在 $\mathrm{CD}$ 上可进行多种 化学修饰; 另外, 在水溶液中良好的溶解性和包结能力 使 $\mathrm{CD}$ 成为环境友好的手性主体分子.

\section{1 基于环糊精的单分子手性光化学反应}

在直链烯氧基苯的间位光环加成反应中，一步反应 可以产生 5 个甚至更多的手性中心. 将 4-苯氧基丁烯衍 生物 1 加入到 $\beta-\mathrm{CD}$ 的热水溶液中可形成化合物 1 与 $\beta$ - CD 的主客体复合物沉淀(Scheme 1) ${ }^{[13]}$. 复合物中主客 体分子的化学计量比跟底物有关, 其中对 $\mathbf{1 b}$ 为 $1: 1$, 对 $1 \mathrm{a}, 1 \mathrm{c}$ 和 $1 \mathrm{~d}$ 为 $2: 1$. 化合物 1 通过光化学反应得到 多环化合物 2 和 3 , 并且对 $1 \mathrm{c}$ 与 $\beta-\mathrm{CD}$ 的固相复合物进 行光照产生的 $2 \mathrm{c}$ 达到 $17 \%$ ee. 在这个体系中是通过 $\beta$-CD 内壁的阻碍作用, 化合物 1 中苯环的一个面被屏 蔽, 从而乙烯基更容易接触苯环相反的面来实现其对映 异构.

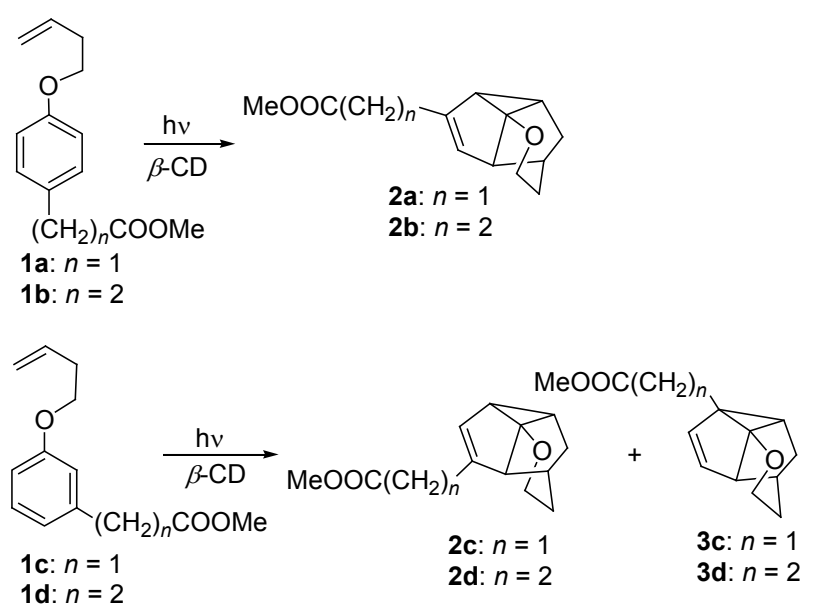

\section{Scheme 1}

对 1,2-二苯基环丙烷衍生物 4 与 $\beta$-CD 形成的复合 物进行直接光照或在光敏剂作用下可以实现 $\mathbf{4}$ 的光异构 化反应(Eq. 1$)^{[14]}$. 通常在固相中进行的光化学反应能够 得到比溶液中进行的光化学反应更高的 $e e$ 值. 化合物 $4 \mathbf{a}$ 与 $\beta$-CD 在固相的复合物与三重态敏化剂 4-甲氧基乙 酰苯机械混和, 通过光敏化反应得到的 $\mathbf{5 a}$ 有 $13 \% e e$ 的 选择性. 该反应的选择性似乎与产物的包结取向有关, 因为与 $\beta$-CD 包结能力更强的 $\mathbf{5 a}$ 对映异构体为过量的对 映异构体 ${ }^{[14]}$.

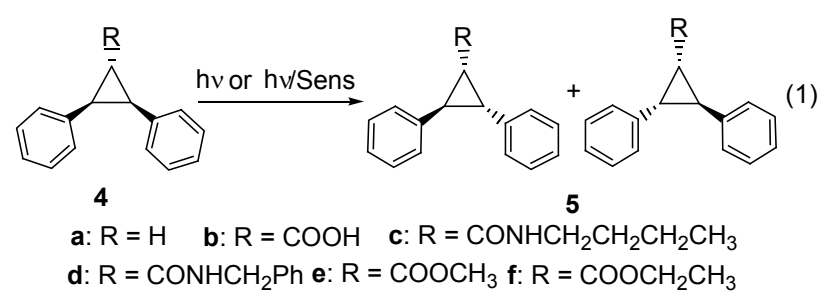

让托酚酮醚 6 (Scheme 2) 在 CD 的水溶液中发生光 环化反应只能得到较低的对映选择性. 然而, 托酚酮 6 与 $\mathrm{CD}$ 的固相复合物的光化学反应其产物 7 的 $e e$ 值可提 高到 33\%. 研究表明, 反应底物与 CD 空腔的尺寸匹配 程度是反应获得高的对映选择性的关键因素，如 $\beta-, \alpha-$ 和 $\gamma$-CD 与底物的匹配程度依次降低, 其光化学反应产 物 7 的 $e e$ 值分别为 $28 \%, 5 \%, 0 \%{ }^{[15]}$.

在吡啶衍生物 8 的光环化反应中(Scheme 2), 固相 中进行的光解反应同样得到比水溶液中反应更高的对 映选择性. 底物 $8 \mathrm{a}$ 和 $8 \mathrm{~b}$ 在水溶液中进行光解时, 使在 $\beta$-CD 大量过量的情况下, 其产物 $e e$ 值也是很低的, 而 对 8 与 $\mathrm{CD}$ 通过机械摚拌混合制备的固相主客体复合物 进行光照时, 则以 $60 \% e e$ 得到产物 $9 \mathbf{b}^{[16]}$. 有趣的是, 固 相复合物中的水分含量对该反应的对映选择性具有很 大影响，当体系中水分含量从 $9 \%$ 降低至 $2 \%$ 时，产物的 $e e$ 从 $60 \%$ 降低至 $26 \%$, 而改变其他不能形成氢键的溶剂 (如正己烷)的含量并没有观察到反应对映选择性较大的 变化. 这种有趣的现象可能是水分子和固相复合物中包 合的客体分子或主体分子 $\mathrm{CD}$ 间的氢键作用的结果.

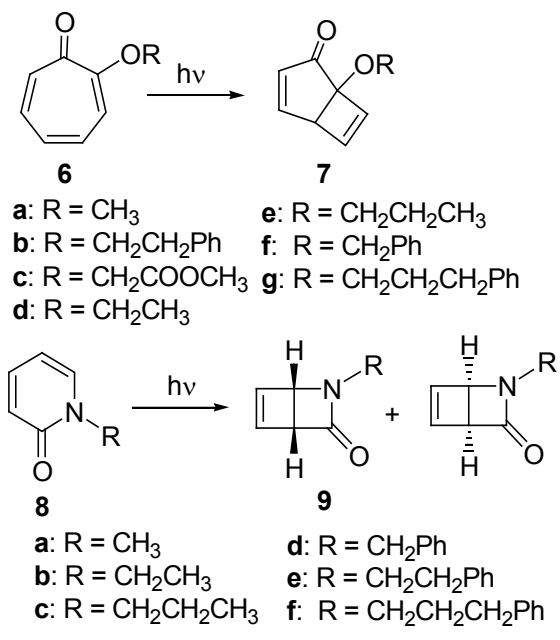

\section{Scheme 2}

对手性的芳基酯 10 (Scheme 3)进行光照，反应在经 历一个螺旋形内酯环过渡态后, 得到脱羧产物 11 并伴 随着 $\mathrm{CO}_{2}$ 的释放 ${ }^{[17-21]}$. 该脱羧反应是一个协同过程. 在 溶液中, 光照手性纯的芳基酯 $\mathbf{1 0}$ 时, 反应过程得到 $>$ 99\% ee 的脱羧产物 11. 而对外消旋化的芳基酯 10 在 CD 溶液中进行光解时, 得到的脱羧产物中, $(R)-11$ 有 


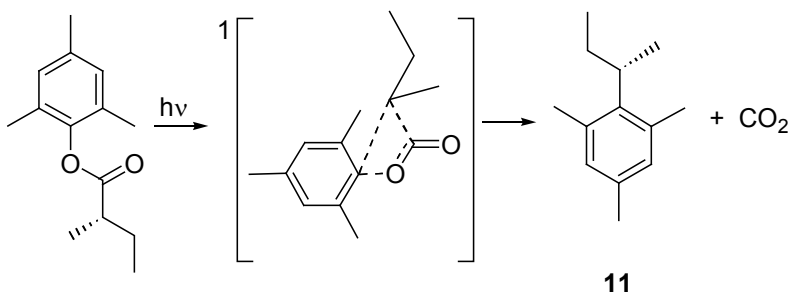

Scheme 3

$14 \%$ 的 $e e$ 值, 说明在 $\beta$-CD 空腔中外消旋芳基酯 10 的其 中一种对映异构体能更快进行光解反应.

Turro 及其合作者 ${ }^{[22]}$ 对苯甲醛 $\mathbf{1 2}$ 的光解反应(Eq. 2) 进行了研究, 得到其双分子结合产物包括 $13 \mathrm{a} \sim$ $13 \mathrm{c}^{[23 \sim 25]}$. 其中, 12 在 $\beta$-CD 的水溶液光解产物 $13 \mathrm{c}$ 达到 $60 \% \sim 70 \%$ 的产率，而产物 13a， 13b 的光解产率分别为 $3 \%$ 和 $<1 \%$. 另一方面, 底物 12 和 $\beta$-CD 形成的 $2: 2$ 固 相复合物以 $70: 30$ 的比例生成 13a 和 13b, 其中手性产 物 13a 有 $15 \%$ ee.<smiles>Cc1ccc(C(O)c2ccccc2C(=O)c2ccccc2C)cc1</smiles><smiles>O=Cc1ccc(C(=O)c2cc[c-]cc2)cc1</smiles>

\section{2 基于环糊精的双分子手性光化学反应}

蒽酸的 $[4+4]$ 光二聚反应(Eq. 3 )是超分子手性光化 学反应中最具代表性的反应之一. 该反应在不同超分子 体系中被研究和验证. 1984 年 Tamaki 及其合作 者 ${ }^{[26,27]}$ 研究了葱酸在 $\gamma$-CD 存在下的光二聚反应, 他们 发现 $\gamma-\mathrm{CD}$ 能够明显加快反应速率, 并且加入 $\gamma-\mathrm{CD}$ 可将 该反应的量子产率从 0.05 提高到 0.4. 通常情况下该反 应产物为 9,10-桥连的光二聚产物 14 17, 其中顺-头对 尾 $(\mathrm{HT})$ 二聚体 15 和反-头对头 $(\mathrm{HH})$ 二聚体 16 具有手

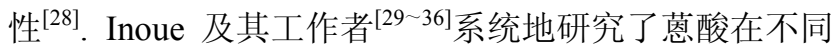
手性主体分子诱导下的光二聚反应的手性选择性, 并且 通过 HPLC 分离得到各个立体异构体. 天然 $\gamma$-CD 可与 客体分子形成 $1: 2$ 的主客体复合物, 在 $25{ }^{\circ} \mathrm{C}$ 水溶液中 其络合常数分别为 $K_{1}=161 \mathrm{~L} \cdot \mathrm{mol}^{-1}, K_{2}=38500 \mathrm{~L} \cdot$ $\mathrm{mol}^{-1}$. 葱酸与天然 $\gamma-\mathrm{CD}$ 的光二聚反应中, 在 $0{ }^{\circ} \mathrm{C}$ 下得 到 HT 二聚体 15 的 $e e$ 值为 $41 \%$, 但 $\mathrm{HH}$ 二聚体 $\mathbf{1 6}$ 的 $e e$ 值却 $<5 \%$. 从那以后, 多个课题组相继合成了一系列 经修饰的 $\gamma-\mathrm{CD}$ 用于提高葱酸光二聚反应的立体选择性.

为了阐明 $\mathrm{CD}$ 骨架的改变对该光二聚反应立体选择

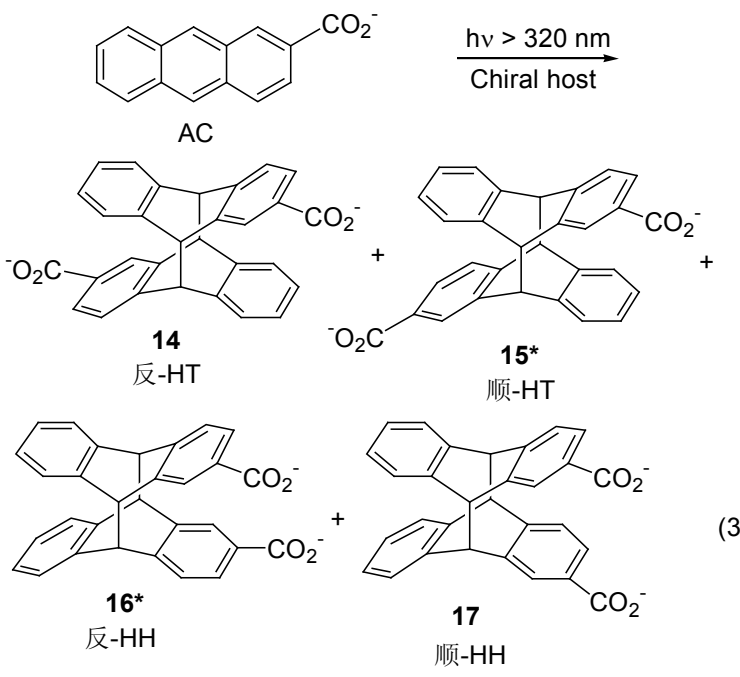

性的影响，我们合成了几种第二面修饰的 $\mathrm{CD}$ 衍生物

18a 18e 来作为主体分子(Scheme 4). 以这些被修饰的 $\mathrm{CD}$ 衍生物参与的反应主要生成了二聚体 $\mathbf{1 5}$, 其 $e e$ 值与 天然 $\mathrm{CD}$ 的结果相当或更低. 然而，带有胺基的 $18 \mathrm{e}$ 作

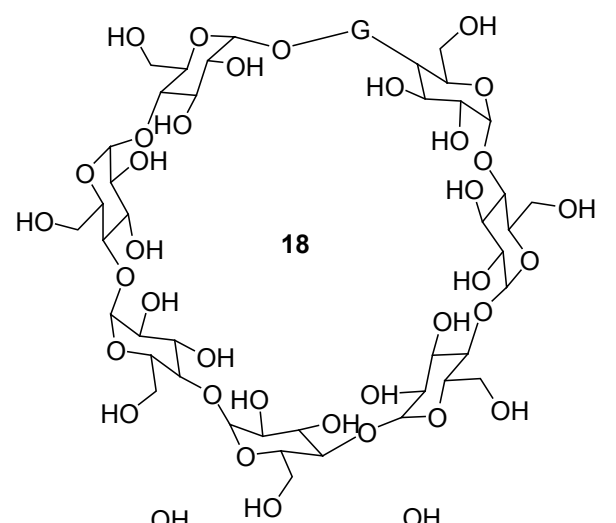

G:
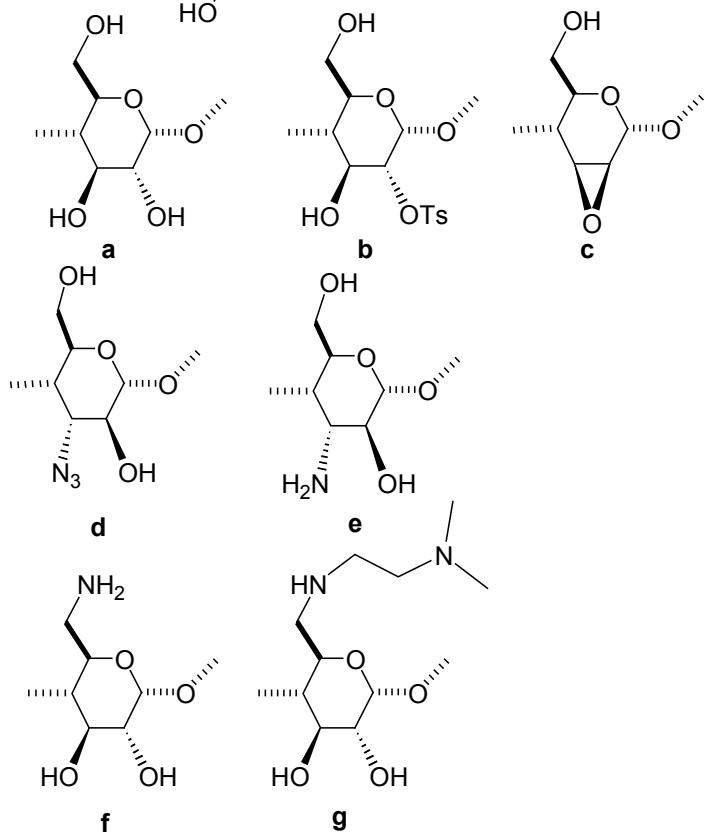

Scheme 4 
为主体分子时明显提高了光二聚产物 $\mathbf{1 5}$ 的 $e e$ 值, 这是 胺基正离子和羧基负离子的静电作用以及阿卓糖结构 单元致使 $\mathrm{CD}$ 的空腔变形双重作用的结果 ${ }^{[3]}$. 改变反应 液的温度和压力可使反应的立体选择性发生转变. 提高 反应体系的压力不仅可区分中间体或过渡态复合物的 体积差异, 也可降低体系的冰点. 在 $-21{ }^{\circ} \mathrm{C}$ 和 $210 \mathrm{MPa}$ 下, 18e 作为主体分子时葱酸的光二聚反应以 $71 \% e e$ 得 到产物 $\mathbf{1 5}$.

当一面上具有两个阳离子型取代基的 $\gamma-\mathrm{CD}$ 衍生物 18g, 19a 19d 和 20a 20d 作为主体分子时(Scheme 5), 可通过一面上的两个阳离子与包结的葱酸分子对上的 羧基间的静电作用来提高 $\mathrm{HH}$-二聚体的立体选择 性 ${ }^{[29,30]}$. HH-二聚体的产率得到增加, 在优化了的温度 和溶剂条件下, 以 20c 作为主体分子得到 $22 \% e e$ 的 HH二聚体 16, 而以 $20 \mathrm{~b}$ 为主体分子时产物 $e e$ 值为 $35 \%$. 对 于跨环的二胺基 $\gamma-\mathrm{CD}$ 衍生物 19a $~ 19 d$, 反-HH 二聚体 16 与顺- $\mathrm{HH}$ 二聚体 17 的相对产率明显随环上两个胺基 间距离的变化而改变. 随着 $\mathrm{CD}$ 环上两个胺基距离的增

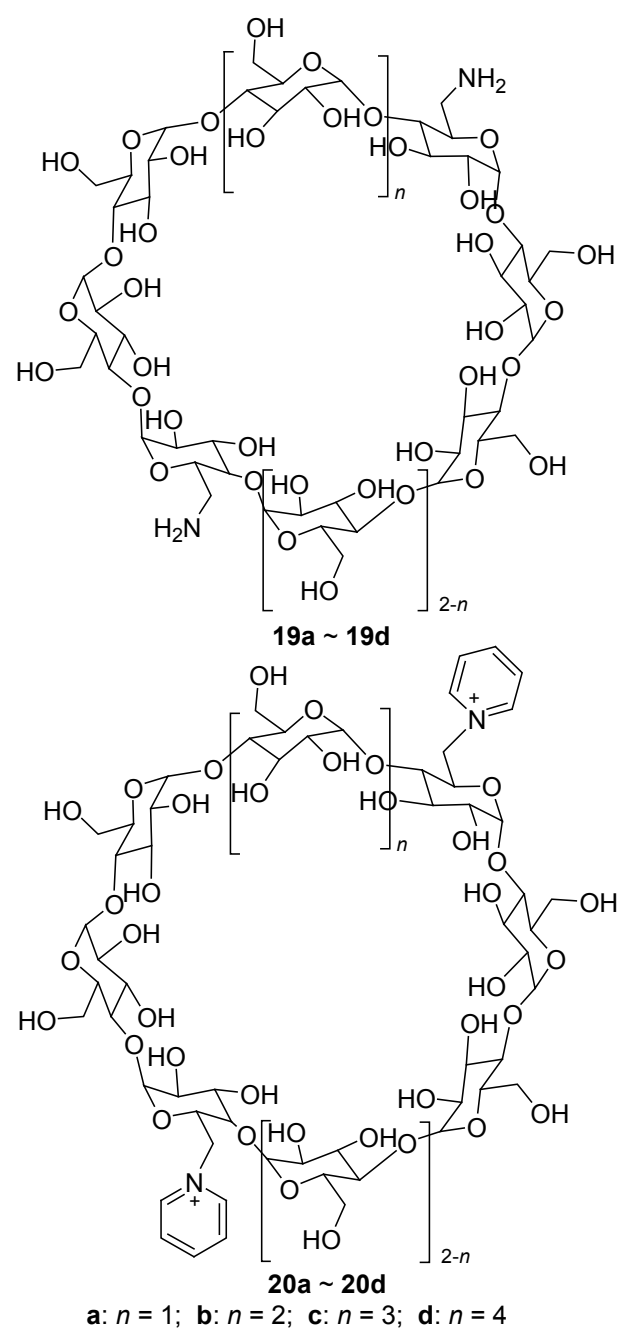

Scheme 5
加，二聚产物 16/17 的比率明显提高. 具有两个阳离子 型取代基的 $18 \mathrm{~g}$ 作为主体分子时, 在低温的甲醇水溶液 中, 反应产物 17 的 $e e$ 值达到 41\%, 远高于 19a 19d 作 为主体分子时的 $e e$ 值, 表明 $\mathrm{CD}$ 环上具有柔性侧臂对提 高反应的对映选择性具有更好的效果.

几种盖帽 $\gamma$-CD 21 25 (Scheme 6) 和二胺基 $\gamma-\mathrm{CD}$ 26 28 也被用于葱酸的光二聚反应 ${ }^{[2,38]}$. 与天然 $\gamma-C D$ 相比，盖帽 CD 22 和 23 明显降低了产物 15 的对映选择 性 ${ }^{[32]}$. 相反，具有一个更加刚性的盖帽的主体分子 21 使产物 15 的对映选择性发生倒转, 并且将其 $e e$ 值提高 到 $-56 \%$. 由于主体分子 21 和 22 的结构上仅仅只有一 个氧原子的微小差别, 对反应的对映选择性却表现出巨 大的差异，这表明了在超分子体系中结构和功能间的紧 密关联. $\gamma$-CDs 24 和 25 (Scheme 6) 的紫外, 圆二色以及 荧光光谱均表现出明显的 $\mathrm{pH}$ 依赖性. 它们作为葱酸光 二聚反应的主体时, 其对映选择性也受到 $\mathrm{pH}$ 影响. 改 变溶液的 $\mathrm{pH}$ 值甚至可以引起 $\mathrm{HH}$-二聚产物 $\mathbf{1 6}$ 的 $e e$ 值 发生逆转, 这是由于其盖帽基团会随着 $\mathrm{pH}$ 的变化发生 构象上的改变造成的. 和修饰的 CD 19 和 20 相类似，二 胺基修饰的 $\gamma$-CD 26 28 能有效地提高 $\mathrm{HH}$ 二聚体的产 率(Scheme 7) ${ }^{[38]}$.

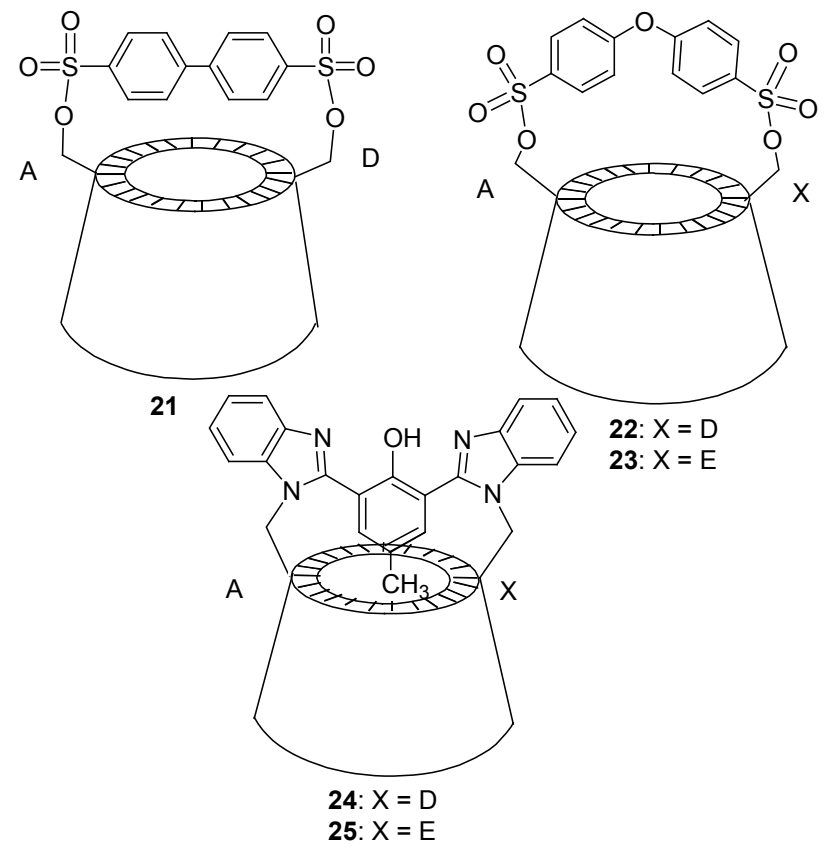

Scheme 6

我们将一个或两个葱酸分子共价连接到 $\alpha-\mathrm{CD}$ 上来 研究葱酸光二聚反应时多重超分子作用对反应立体化 学的影响(Scheme 8). 让 $\alpha-\mathrm{CD}$ 衍生物 29 和 30a $\sim 30 \mathrm{c}$ 在 $\gamma-\mathrm{CD}$ 或葫芦腿 $[8]$ 的空腔中发生光化学反应并对产物进 行水解得到 14 17. 在 $210 \mathrm{MPa}$ 以及 $-20{ }^{\circ} \mathrm{C}$ 下底物 29 在 $\gamma-\mathrm{CD}$ 中发生光解，以 $68 \%$ 的产率和 $91 \% e e$ 得到产物 

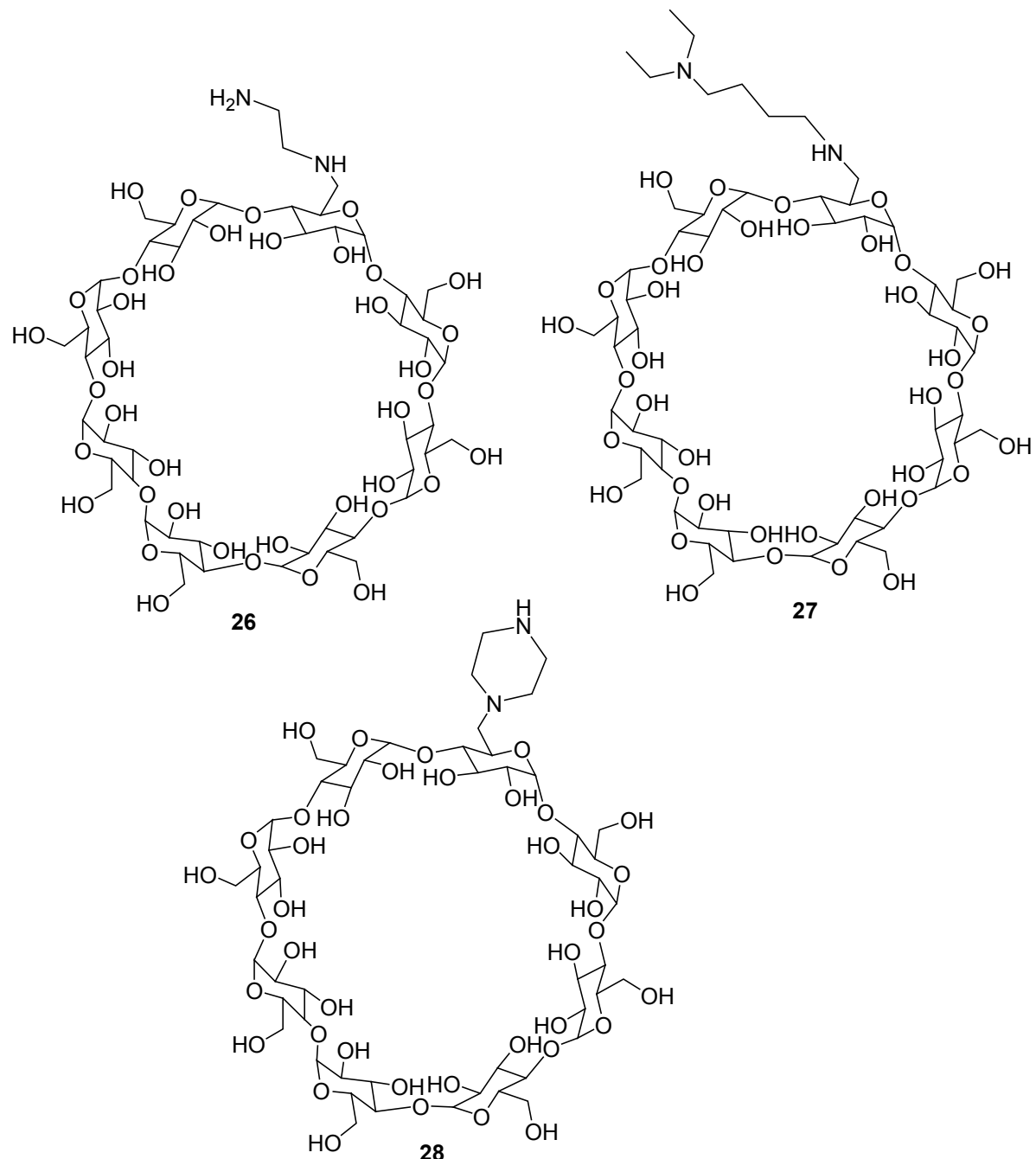

Scheme 7
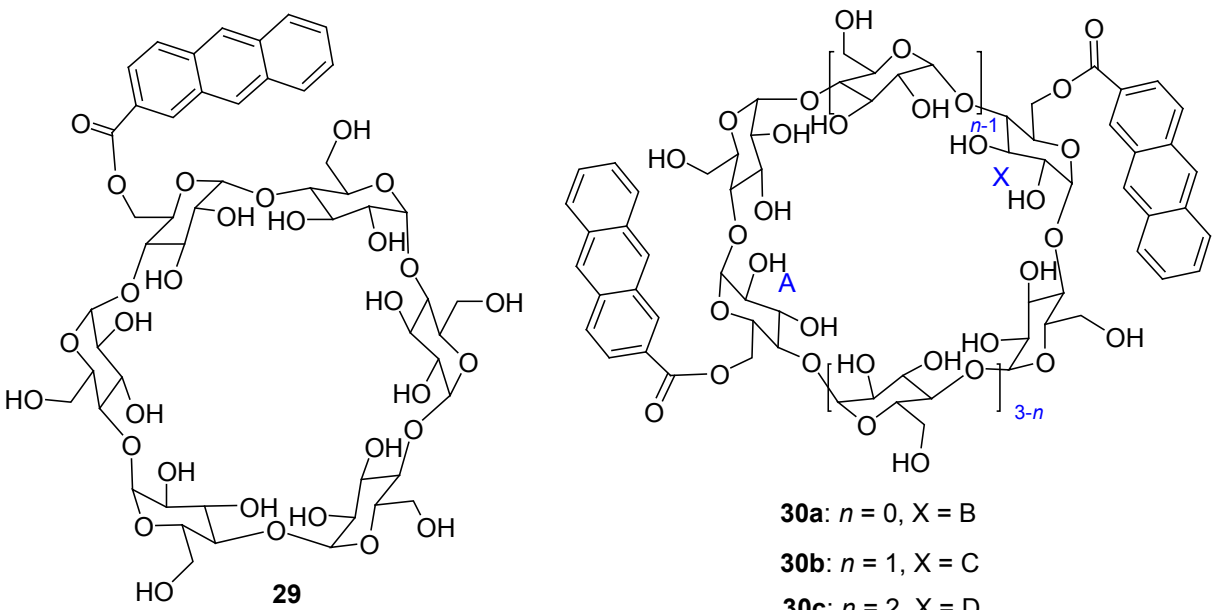

30a: $n=0, X=B$

30b: $n=1, \mathrm{X}=\mathrm{C}$

30c: $n=2, X=\mathrm{D}$

Scheme 8

15. 有趣的是在葫芦嫝 [8] 空腔中反应时, 得到 $\mathrm{HH}$ 二聚 体 16 和 17 为主要产物且产率共达到 99\%, 这是由于葫 芦腿的空腔相对较长, 在 $\alpha$ - $\mathrm{CD}$ 的立体阻碍下葫芦艮空 腔中 HT 取向的两个葱酸分子的 9,10 位无法获得良好的
匹配. 当底物 $30 \mathrm{a} \sim 30 \mathrm{c}$ 被光解时，其反应产物随二曾酸 的位置不同表现出巨大差异. 对底物 $30 \mathrm{a} \sim 30 \mathrm{~b}$ 进行光 解几乎完全生成 HH-二聚体, 而底物 30c 则主要生成 HT 二聚体. 对底物 30b 和 30c 进行光解可分别得到 $92 \% e e$ 
的 16 和 $63 \% e e$ 的 15 . 当反应在 $\gamma-\mathrm{CD}$ 或葫芦腿 [8]媒介 中进行时, 控制在最优化的反应条件下可以以 $98 \%$ 的产 率和 $99 \% e e$ 得到产物 $\mathbf{1} 7^{[39]}$.

最近, 研究发现 9-羟基葱(HA)在 $\gamma$-CD 媒介中发生 的光二聚反应(Eq. 4), 并可得到四种产物 31 34. HA 的 圆二色及吸收光谱研究表明 $\gamma$-CD 与阴离子型的 HA 主 要形成 $1: 1$ 的复合物, 其络合常数为 $(4100 \pm 390) \mathrm{L} \cdot$ $\mathrm{mol}^{-1}$, 这可能是由于 $\mathrm{HA}$ 分子局部的负电荷排斥作用 阻碍了第二个 HA 阴离子与 $\gamma-\mathrm{CD}$ 络合. 而中性的 HA 分 子可以与 $\gamma-\mathrm{CD}$ 形成稳定 $1: 2$ 主客体复合物. 溶液中在 $\gamma$-CD 存在下进行的 HA 分子的光解反应, 以 $12 \% \sim 14 \%$ $e e$ 得到顺-HT 产物 32, 以 $5 \% \sim 6 \% e e$ 得到反-HH 产物 33. 在通过 HA 与 0.5 equiv. 的 $\gamma-\mathrm{CD}$ 进行碾磨形成的固 相复合物中进行光照反应, 以 $48 \%$ 产率得到反-HH 产物 33 的外消旋体, 而顺-HT 产物 32 具有 $-17 \% e e^{[40]}$.

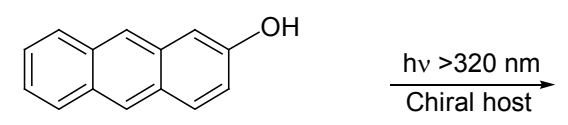

$\mathrm{HA}$
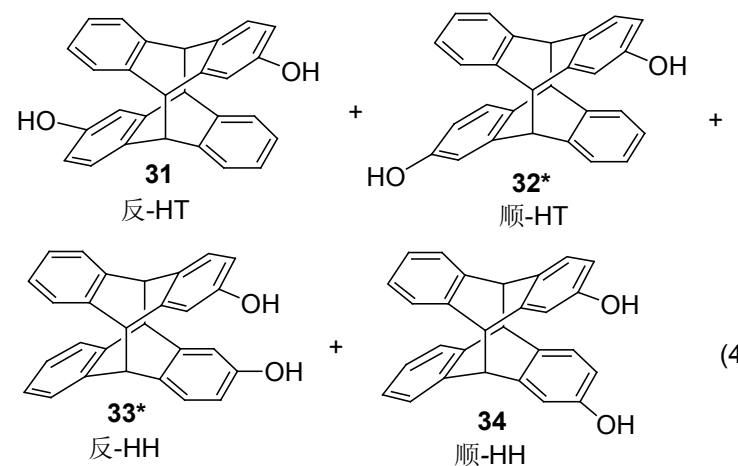

$\mathrm{Wu}$, Tong 及合作者 ${ }^{[41,42]}$ 研究了 3-甲基-2-萗酯(35) 在 $\gamma$-CD 中进行的光二聚反应(Scheme 9)及其对映选择 性. 他们发现底物 35 发生光二聚反应生成 37 是一个双 光子吸收过程, 当底物吸收第一个光子时生成 $[4+4]$ 环 加成产物 36, 进而吸收第二个光子转变为光二聚产物 37. 天然 $\gamma-C D$ 与底物 35 具有非常强的结合能力, 其键 合常数达到 $6.7 \times 106\left(\mathrm{~mol} \cdot \mathrm{L}^{-1}\right)^{-2}$. 底物 35 与 $\gamma-\mathrm{CD}$ 形成 的 $2: 1$ 的复合物明显提高了其光二聚反应的效率, 而 在没有 $\gamma-\mathrm{CD}$ 的水溶液中该反应几乎是不进行的. $\gamma-\mathrm{CD}$ 在反应中对中间产物 36 和 37 具有不同程度的手性诱导 作用, 其中前者具有 $39 \%$ ee 而后者具有 $48 \% \mathrm{ee}$. 对于 那些在 $\gamma-\mathrm{CD}$ 二面上修饰的主体分子 $18 \mathrm{~b} \sim 18 \mathrm{e}$ 对反应的 对映选择性仅有轻微减少 ${ }^{[43]}$.

\section{3 基于环糊精的手性光催化反应}

天然 $\mathrm{CD}$ 曾经被用来研究光诱导的 $(Z)$-环辛烯 $38 \mathrm{Z}$ 到面手性 $(E)$-环辛烯 $38 \mathrm{E}$ 转变的不对称光异构化反应

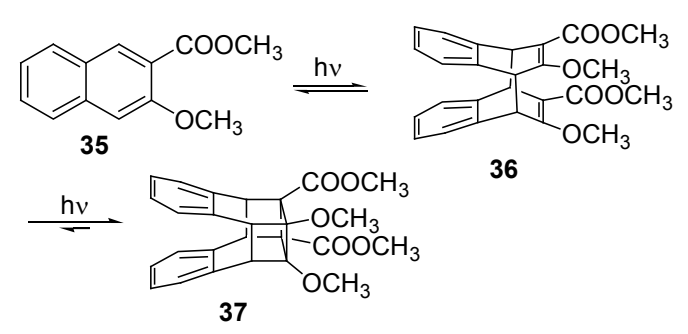

Scheme 9

(Scheme 10). 天然 $\mathrm{CD}$ 与 $\mathbf{3 8 Z}$ 形成 $1: 1$ 的主客体复合 物而从水溶液中沉淀出来. 固态复合物在 $185 \mathrm{~nm}$ 的直 接激发下仅产生很小的 $e e$ 值, 表明出天然 $\beta$-CD 对 $\mathbf{3 8 Z}$ 有弱的不对称诱导能力 ${ }^{[44]}$. Inoue 和同事 ${ }^{[7,45 \sim 51]}$ 探索研究 了在一系列苯甲酸酯修饰的 CD 包结和敏化作用下 $\mathbf{3 8 Z}$ 的超分子光异构化反应(Scheme 11). 如 Scheme 11 所示, 在没有客体分子的情况下，敏化剂部分由于疏水作用而 呈一种自包结构象, 在 CD 空腔的保护下不能敏化溶液 中的底物. 然而, 当 $\mathbf{3 8 Z}$ 与敏化剂修饰的 $\mathrm{CD}$ 复合时, 敏 化剂部分会在一定程度上被推出去, 由于空间上的邻 近，敏化剂激发态的能量会有效地传递给 38Z. 当使用 40b 作为敏化剂时, 38E 的 $e e$ 值显著地增加到 $24 \%$. 有 趣的是，使用 $40 \mathrm{~g}$ 时 $38 \mathrm{Z}$ 光异构化反应显示出高达 $46 \%$ 的 $e e$ 值 ${ }^{[45,49]}$, 而邻位和对位异构体 $40 \mathrm{f}$ 和 $40 \mathrm{~h}$ 只有很弱 的对映选择性. 这种由主体结构上微小的变化产生的不 同立体化学结果, 显示出化学修饰对于超分子体系光手 性源的研究有着很大的潜力 ${ }^{[49]}$.

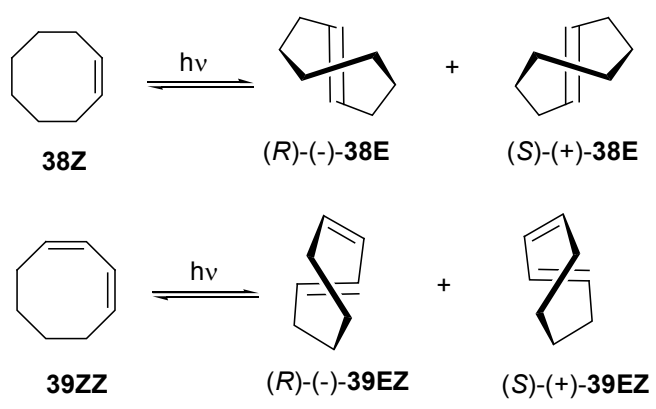

Scheme 10

使用传统的手性敏化剂, $38 \mathrm{Z}$ 的光异构化反应很容 易受到熵相关因素比如温度、溶剂和压力的影响 $\left.{ }^{[52} 56\right]$. 相反，基于 $\mathrm{CD}$ 的光敏化剂促进的 $\mathbf{3 8 Z}$ 光异构化反应的 对映选择性几乎不由熵决定, 因为 $\mathrm{CD}$ 空腔相对刚性的 性质, 改变温度没有对 $e e$ 造成明显的变化. 然而, 在敏 化剂 40i 包结下, $38 \mathrm{Z}$ 光异构化反应中观察到了由温度 改变引起的对映选择性的明显改变, 这是由于 $40 \mathrm{i}$ 的骨 架因 CD 第二面的氢键网络的破坏而变得柔软 ${ }^{[26,28]}$.

对于 $(Z, Z)-1,3$-环辛烯 $39 \mathrm{ZZ}$ 转变为手性 $(E, Z)-1,3$-环 辛二烯 39EZ 的光异构化反应(Scheme 10), 其对应异构 选择性比其同系物 $39 \mathrm{Z}$ 要困难很多. 用传统的敏化剂 

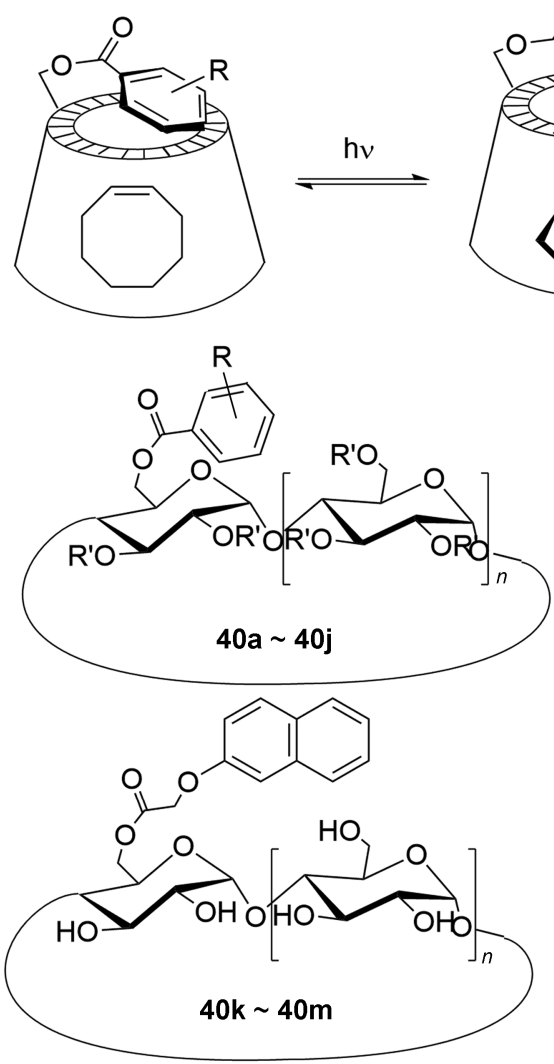

40a: $\mathrm{R}=\mathrm{H}, n=5, \mathrm{R}^{\prime}=\mathrm{H}$

40b: $\mathrm{R}=o-\mathrm{OMe}, n=6, \mathrm{R}^{\prime}=\mathrm{H}$

40c: $\mathrm{R}=\mathrm{O}-\mathrm{CO}_{2} \mathrm{Me}, n=6, \mathrm{R}^{\prime}=\mathrm{H}$

40d: $\mathrm{R}=m-\mathrm{CO}_{2} \mathrm{Me}, n=6, \mathrm{R}^{\prime}=\mathrm{H}$

40e: $\mathrm{R}=p-\mathrm{CO}_{2} \mathrm{Me}, n=6, \mathrm{R}^{\prime}=\mathrm{H}$

40f: $\mathrm{R}=0-\mathrm{OMe}, n=6, \mathrm{R}^{\prime}=\mathrm{H}$

40g: $\mathrm{R}=m$-OMe, $n=6, \mathrm{R}^{\prime}=\mathrm{H}$

40h: $\mathrm{R}=p$-OMe, $n=6, \mathrm{R}^{\prime}=\mathrm{H}$

40i: $\mathrm{R}=\mathrm{H}, n=6, \mathrm{R}^{\prime}=\mathrm{Me}$

40j: $\mathrm{R}=\mathrm{H}, n=7, \mathrm{R}^{\prime}=\mathrm{H}$

40k: $n=5(\alpha-C D)$

40I: $n=6(\beta-C D)$

40m: $n=7(\gamma-\mathrm{CD})$
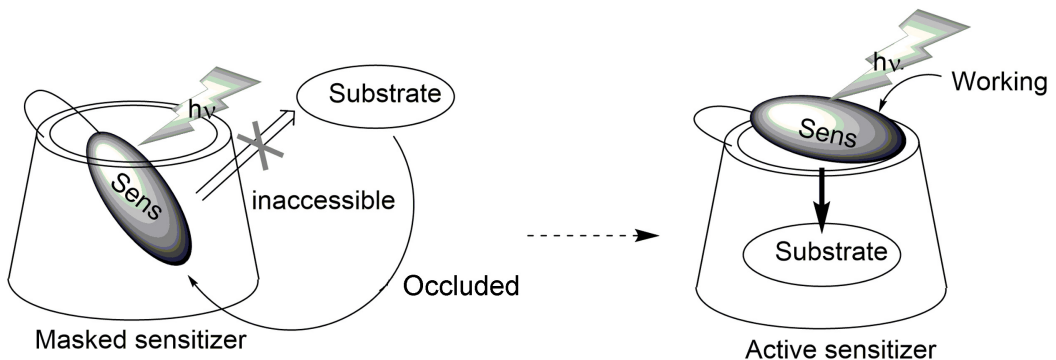

Scheme 11

苯六甲酸薄荷酯敏化, 在 $-40{ }^{\circ} \mathrm{C}$ 的低温下反应得到最 高 $17 \%$ 的 $e e$ 值 ${ }^{[57]}$. 以菜的 $\mathrm{CD}$ 衍生物 $\mathbf{4 0 k} \sim 40 \mathrm{~m}$ 作为超 分子敏化剂, 我们考察了 $39 \mathrm{ZZ}$ 的光敏化异构化反应. 在 $\beta-\mathrm{CD}$ 衍生物 401 的水溶液中, 获得了 $4.6 \% e e^{[58]}$.

最近, 一系列新颖的基于 $\mathrm{CD}$ 的超分子结构, 即 $\mathrm{CD}$ 微海绵体(CDNS) 41 43 (Scheme 12) ${ }^{[39,40]}$, 被用于研究 $38 \mathrm{Z}$ 和 $39 \mathrm{ZZ}$ 的光异构化反应. CDNS $41 \sim 43$ 由 $2 \sim 4$ equiv. 的均苯四甲酸二䣶(PDA)与 $\beta$-CD 和 $\gamma$-CD 反应而 成. 将 CDNS 的浓度从 $0.2 \mathrm{mg} / \mathrm{mL}$ 逐渐增大至 2000 $\mathrm{mg} / \mathrm{mL}$, 可产生一系列相的改变: 溶液中先产生沉淀, 而后变为流动的凝胶, 进一步提高 CDNS 浓度形成了刚 性的凝胶. CDNS 作用下时 $\mathbf{3 9 Z Z}$ 超分子光异构化反应 容易受 CDNS 的相转变影响. 例如, 由 $\beta$-CDNS 41 敏化 的 $39 \mathrm{ZZ}$ 的光异构化反应中, $39 \mathrm{EZ}$ 的 $e e$ 值随着相态由溶
液转变为悬浮物, 产物的对应异构选择性从 $4.7 \% \mathrm{ee}$ 下 降为 $0 \% \mathrm{ee}$, 但是在刚性凝胶部分, 又上升到了 $6.1 \% \mathrm{ee}$. 由 43 敏化的 $39 Z Z$ 的光异构化反应中, 在溶液和流动的 凝胶态中得到的几乎是外消旋的产物, 而在凝胶态中 39ZZ 获得了目前的超分子光异构化反应观察到的最高 的 $13.3 \%$ ee 的选择性.

在用 CDNS 敏化的 $\mathbf{3 8 Z}$ 的光异构化反应中也观察 到了相同的相态依赖的对映选择性，随着 CDNSs 相态 的改变, 其相关的手性环境的改变, 被认为是相态控制 的光手性产生的原因.

在葱酸的光二聚反应体系中，我们利用二氨基修饰 的 $\gamma$-CDs 26 28 (Scheme 7) 实现了一种与常规的光敏化 方法不同的手性光催化方法, $\gamma-\mathrm{CD}-\mathrm{Cu}^{2+}$ 复合物作为手 性主体分子时，在 $-50{ }^{\circ} \mathrm{C}$ 的甲醇水溶液中, 0.1 equiv. $\gamma$ - 


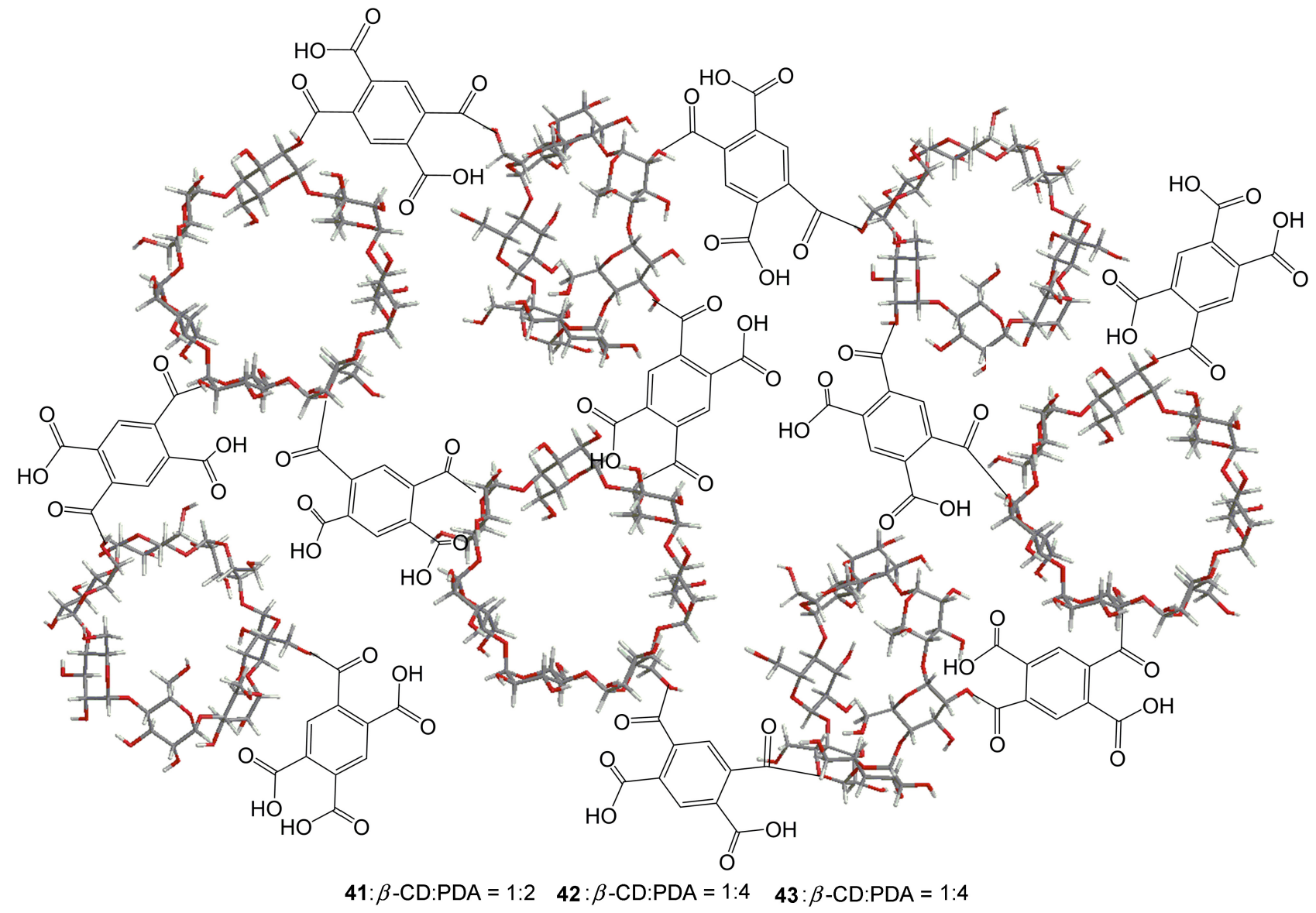

Scheme 12

$\mathrm{CD}-\mathrm{Cu}^{2+}$ 复合物存在时, 产物 $\mathrm{HH}$ 二聚产物 16 能够达到 $52 \%$ 产率以及 $70 \% \mathrm{ee}$. 这一非敏化光催化是由 $\mathrm{Cu}^{2+}$ 的配 位作用以及 $\mathrm{CD}$ 空腔外金属离子的抑制作用共同实现 的.

\section{4 基于环糊精手性光化学反应的波长效应}

用 $\gamma-\mathrm{CD}$ 包结蒽酸分子所进行的光二聚反应为实现 反应的立体选择性提供了一种新的手段, 即波长调控手 段. 该想法来源于 $\gamma-\mathrm{CD}$ 与蒽酸形成的 $1: 2$ 复合物对不 同波长的光的吸收存在差异. 如 Scheme 13 所示, 有三 个关键的步骤决定了蒽酸的光二聚反应的立体选择性, 即包结, 光激发及化学转化过程. 蒽酸在水溶液中的激 发态寿命为 $16 \mathrm{~ns}$, 在这段极短的时间内, 葱酸和 $\gamma-\mathrm{CD}$ 形成的 $1: 2$ 复合物相互之间无法形成转换, 因此, 二聚 体产物的相对产量本质上与相应的受到激发的前体复 合物成比例, 即呈 HT-方向排列的一对蒽酸分子将形成 HT 二聚体产物, 相应的, 呈 HH-方向排列的一对葱酸 分子将形成 $\mathrm{HH}$ 二聚体产物. 由于前体复合物有着不同 的吸收光谱, 它们受到不同波长光激发的概率将随着激 发波长的变化而变化. 事实证明, 反应的立体选择性确 实随着激发波长的变化发生了显著地改变. 借助 $\gamma-\mathrm{CD}$
的包结作用，二聚体 $\mathbf{1 4}$ 和 $\mathbf{1 5}$ 在 $300 \mathrm{~nm}$ 的激发波长下的 产率分别 $54.5 \%$ 和 $33.3 \%$, 而在 $440 \mathrm{~nm}$ 的激发波长下, 二者的产率变为 $30.7 \%$ 和 $60.8 \%$, 并且, 改变激发波长 会造成 $e e$ 值发生很大的波动，使得 15 和 16 的 $e e$ 值分 别在 $24 \%$ 到 $41 \%$ 和 $-8.8 \%$ 到 $11.9 \%$ 间波动. 另一方面, 波长效应受到手性主体分子的化学结构，反应温度和溶 剂的共同影响，说明这些外界因素对于客体蒽酸分子的 几何堆积方式均有着重要的影响 ${ }^{[59]}$.

\section{5 结论及展望}

基于 $\mathrm{CD}$ 的超分子手性光化学合成领域在近年来发 展迅速, 利用 $\mathrm{CD}$ 为手性超分子主体为手性光化学提供 了许多新的可能. 如最近发现的非敏化的手性光催化和 不同的激发波长对反应选择性和光学产率的控制等. CD 由于其易于与客体发生包结作用而被频繁地用作手 性主体，但是通常情况下在 CD 包结下发生光化学反应 的立体选择性并不高. 这意味着在无方向性的疏水作用 主导下的客体分子在 $\mathrm{CD}$ 的轴对称空腔中的手性传递不 是很有效，特别是考虑到 $\mathrm{CD}$ 的内壁对称地分布着 30 个以上的手性中心. 通过修饰或功能化的 $\mathrm{CD}$ 来打破天 然 CD 作为手性主体的局限是一种非常有效的策略. 采 


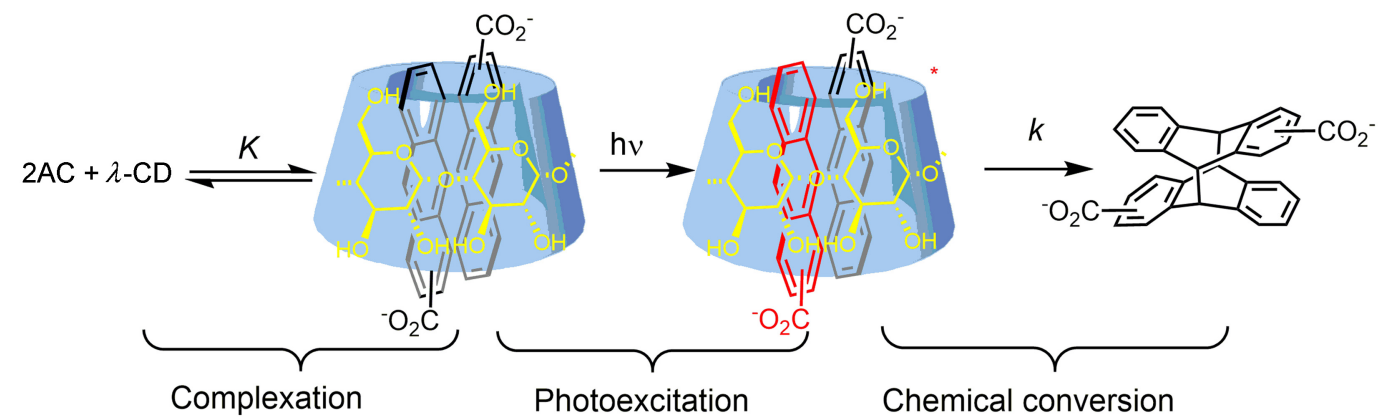

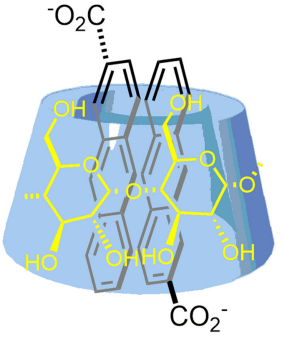

HT Complex

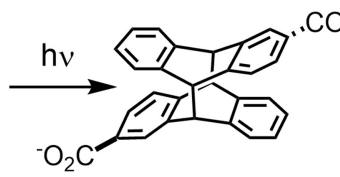

HT dimer

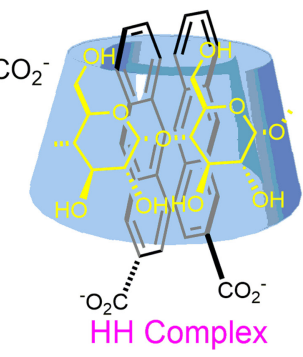

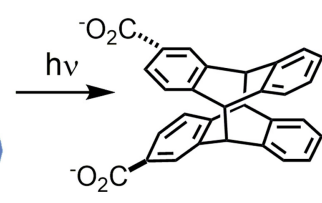

$\mathrm{HH}$ dimer

Scheme 13

用这个方法, $\mathrm{CD}$ 空腔的尺寸、形状、柔软度和不对称环 境都发生了改变. 从而, 反应的化学、区域或立体选择 性通常都能得到提高.

在多数情况下, 在固态中进行的光反应的产率要 高于在溶液中的光反应. 这可以由下述两条理由来解 释: (1)固态的复合物, 特别是共沉淀技术制得的固态复 合物, 几乎所有的客体分子都进入了 $\mathrm{CD}$ 的空腔内. 没 有进入空腔的自由的客体分子在竞争反应中处于不利 的地位, 并且生成的产物都是外消旋的, 因此他们在反 应中的影响可以被排除. (2)液相的 CD 复合物中的客体 分子有更大的位置和转动的自由度, 而在固态复合物中 的客体分子与之相反, 他们在固相 $\mathrm{CD}$ 复合物中严格的 线性排列使得他们在反应中有更高的化学、区域和立体 选择性. 然而, 研究也表明超分子手性光反应体系的柔 软度将会为控制反应产物的 $e e$ 值和手性识别提供新的 机会. 相比于刚性的超分子识别体系通常与高焓值变化 相关, 柔性的主体常伴随着更大的复合物自由度的变 化, 因此更依赖于熵变. 人们可以在不使用相反手性的 引物或主体分子的情况下操控与熵值相关的外部因素, 例如溶剂、温度和压力, 来改变甚至反转反应的立体选 择性, 这将有利于像 CD 这样相反的手性主体不易得到 的情况.

总之基于 $\mathrm{CD}$ 的手性光化学已获得了长足的发展, 为手性光化学提供了独特的手性控制方法. 但其研究的 广度和深度, 特别是在催化手性光化学反应等方面, 有 待进一步提高. 在手性结晶或沸石超分子笼等固相体系 中易实现精确地手性诱导, 而在溶液中进行的手性光催
化反应不易实现高的 $e e$ 值, 使用 $\mathrm{CD}$ 作为手性主体是目 前实现溶液中高效不对称光催化的一个主要策略, 由于 环糊精作为手性主体具有以下优势: (1)环糊精空腔本身 是手性的; (2)连接于环糊精上的光敏剂能促进空腔中底 物与光敏剂本身构象的固定; (3)在未形成超分子络合物 的情况下，光敏剂并不能敏化溶液中的底物，即光敏化 反应只发生在环糊精空腔中. 研究表明，环糊精主体分 子上微小的结构变化能产生不同立体化学结果, 显示了 通过对超分子手性源进行化学修饰来提高手性光反应 $e e$ 值的巨大潜力，该领域的研究有望有效地促进手性光 化学的发展.

\section{References}

[1] (a) Griesbeck, A.G.; Mattay, J. Synthetic Organic Photochemistry, Marcel Dekker, New York, 2005.

(b) Hu, R.; Zhang, C.; Pei, Z. Acta Chim. Sinica 2013, 71, 1064 (in Chinese).

(胡锐, 张承平, 裴志胜, 化学学报, 2013, 71, 1064.)

(c) Tong, L., Lu, R., Inoue, Y. Prog. Chem. 2006, 18, 533 (in Chinese).

(童林荟，鲁润华，井上佳久，化学进展, 2006, 18, 533.)

(d) Lv, F.; Wu, L.; Zhang, L.; Tong, Z.; Zheng, L. Chin. J. Org. Chem. 2006, 26, 599 (in Chinese).

(吕峰峰, 吴骊珠, 张丽萍, 佟振合, 郑利强, 有机化学, 2006, 26, 599.)

(e) Ma, L.; Chen, B.; Wu, L.; Peng, L.; Zhang, L.; Tong, Z. Prog. Chem. 2004, 16, 386 (in Chinese).

(马磊, 陈涁, 吴雨珠, 彭明丽, 张丽萍, 佟振合, 化学进展, 2004, 16, 386.)

(f) Yang, C.; Inoue, Y. Chem. Soc. Rev. 2014, Advance Article, DOI: $10.1039 / \mathrm{C} 3 \mathrm{CS} 60339 \mathrm{C}$

[2] Inoue, Y.; Ramamurthy, V. Chiral Photochemistry, Marcel-Dekker, 
New York, 2004.

[3] Yang, C. Chin. Chem. Lett. 2013, 24, 437.

[4] Yang,C.; Inoue, Y. In Supramolecular Photochemistry: Controlling Photochemical Processes, Eds.: Ramamurthy, V.; Inoue, Y., John Wiley \& Sons, Inc., Hoboken, 2011, p. 115.

[5] Yang, C.; Inoue, Y. In CRC Handbook of Organic Photochemistry and Photobiology, 3rd ed., Vol. 1, Eds.: Griesbeck, A. G.; Oelgemoller, M.; Ghetti, F.; Taylaor \& Francis Group, Boca Raton, 2012, p. 125.

[6] Leibovitch, M.; Olovsson, G.; Sundarababu, G.; Ramamurthy, V.; Scheffer, J. R.; Trotter, J. J. Am. Chem. Soc. 1996, 118, 1219.

[7] Inoue, Y.; Dong, S. F.; Yamamoto, K.; Tong, L.-H.; Tsuneishi, H.; Hakushi, T.; Tai, A. J. Am. Chem. Soc. 1995, 113, 2793.

[8] Bach, T.; Bergmann, H.; Harms, K. Angew. Chem., Int. Ed. 2000, 39, 2302.

[9] Bach, T.; Bergmann, H.; Grosch, B.; Harms, K. J. Am. Chem. Soc. 2002, 124, 7982.

[10] shida, Y.; Kai, Y.; Kato, S.-Y.; Misawa, A.; Amano, S.; Matsuoka, Y.; Saigo, K. Angew. Chem., Int. Ed. 2008, 47, 8241.

[11] (a) Shirakawa, M.; Fujita, N.; Tani, T.; Kaneko, K.; Shinkai, S. Chem. Commun. 2005, 4149.

(b) Yin, Y.; Jie, J.; Diao, G. Chemistry 2009, 72, 320 (in Chinese). (殷亚星, 解菊, 刀国旺, 化学通报, 2009, 72, 320.)

[12] Takahashi, K. Chem. Rev. 1998, 98, 2013.

[13] Vizvardi, K.; Desmet, K.; Luyten, I.; Sandra, P.; Hoornaert, G.; Van der Eycken, E. Org. Lett. 2001, 3, 1173.

[14] Koodanjeri, S.; Ramamurthy, V. Tetrahedron Lett. 2002, 43, 9229.

[15] Koodanjeri, S.; Joy, A.; Ramamurthy, V. Tetrahedron 2000, 56, 7003.

[16] Shailaja, J.; Karthikeyan, S.; Ramamurthy, V. Tetrahedron Lett. 2002, 43, 9335.

[17] Mori, T.; Wada, T.; Inoue, Y. Org. Lett. 2000, 2, 3401.

[18] Mori, T.; Inoue, Y.; Weiss, R. G. Org. Lett. 2003, 5, 4661.

[19] Mori, T.; Weiss, R. G.; Inoue, Y. J. Am. Chem. Soc. 2004, 126, 8961.

[20] Mori, T.; Saito, H.; Inoue, Y. Chem. Commun. 2003, 2302.

[21] Mori, T.; Takamoto, M.; Wada, T.; Inoue, Y. Photochem. Photobiol. Sci. 2003, 2, 1187.

[22] Rao, V. P.; Turro, N. J. Tetrahedron Lett. 1989, 30, 4641.

[23] Closs, G. L.; Paulson, D. R. J. Am. Chem. Soc. 1970, 92, 7229.

[24] Cocivera, M.; Trozzolo, A. M. J. Am. Chem. Soc. 1970, 92, 1772.

[25] Berger, M.; Goldblatt, I. L.; Steel, C. J. Am. Chem. Soc. 1973, 95, 1717.

[26] Tamaki, T.; Kokubu, T. J. Inclusion Phenom. Macrocyclic Chem. 1984, 2,815 .

[27] Tamaki, T.; Kokubu, T.; Ichimura, K. Tetrahedron Lett. 1987, 43, 1485.

[28] Wakai, A.; Fukasawa, H.; Yang, C.; Mori, T.; Inoue, Y. J. Am. Chem. Soc. 2012, 134, 4990.

[29] Ikeda, H.; Nihei, T.; Ueno, A. J. Org. Chem. 2005, 70, 1237.

[30] Yang, C.; Fukuhara, G.; Nakamura, A.; Origane, Y.; Fujita, K.; Yuan, D.-Q.; Mori, T.; Wada, T.; Inoue, Y. J. Photochem. Photobiol. A: Chem. 2005, 173, 375.

[31] Yang, C.; Nakamura, A.; Fukuhara, G.; Origane, Y.; Mori, T.; Wada, T.; Inoue, Y. J. Org. Chem. 2006, 71, 3126.

[32] (a) Yang, C.; Nakamura, A.; Wada, T.; Inoue, Y. Org. Lett. 2006, 8, 3005 . (b) Yang, C.; Mori, T.; Inoue, Y. J. Org. Chem. 2008, 73, 5786.

[33] Yang, C.; Ke, C.; Fujita, K.; Yuan, D.-Q.; Mori, T.; Inoue, Y. Aust. J. Chem. 2008, 1.

[34] Ke, C.; Yang, C.; Mori, T.; Wada, T.; Liu, Y.; Inoue, Y. Angew. Chem., Int. Ed. 2009, 48, 6675.

[35] Ke, C.; Yang, C.; Liang, W.; Mori, T.; Liu, Y.; Inoue, Y. New J. Chem. 2010, 34, 1323.

[36] Wang, Q.; Yang, C.; Fukuhara, G.; Mori, T.; Liu, Y.; Inoue, Y. Beilstein J. Org. Chem. 2011, 7, 290.

[37] Yang, C.; Nakamura, A.; Fukuhara, G.; Origane, Y.; Mori, T.; Wada, T.; Inoue, Y. J. Org. Chem. 2006, 71, 3126.

[38] Yang, C.; Ke, C.; Kahee, F.; Yuan, D.-Q.; Mori, T.; Inoue, Y. Aust. J. Chem. 2008, 61, 565.

[39] Ke, C.; Yang, C.; Mori, T.; Wada, T.; Liu, Y.; Inoue, Y. Angew. Chem., Int. Ed. Engl. 2009, 48, 6675.

[40] Fukuhara, G.; Umehara, H.; Higashino, S.; Nishijima, M.; Yang, C.; Mori, T.; Wada, T.; Inoue, Y. Photochem. Photobiol. Sci. 2013.

[41] Liao, G.-H.; Luo, L.; Xu, H.-X.; Wu, X.-L.; Lei, L.; Tung, C.-H.; Wu, L.-Z. J. Org. Chem. 2008, 73, 7345.

[42] Luo, L.; Cheng, S.-F.; Chen, B.; Tung, C.-H.; Wu, L.-Z. Langmuir 2010, 26, 782 .

[43] Liang, W.; Zhang, H.-H.; Wang, J.-J.; Peng, Y.; Chen, B.; Yang, C.; Tung, C.-H.; Wu, L.-Z.; Fukuhara, G.; Mori, T.; Inoue, Y. Pure Appl. Chem. 2011, 83, 769 .

[44] Inoue, Y.; Kosaka, S.; Matsumoto, K.; Tsuneishi, H.; Hakushi, T.; Tai, A.; Nakagawa, K.; Tong, L. J. Photochem. Photobiol. A: Chem. 1993, 71, 61.

[45] Inoue, Y.; Wada, T.; Sugahara, N.; Yamamoto, K.; Kimura, K.; Tong, L.-H.; Gao, X.-M.; Hou, Z.-J.; Liu, Y. J. Org. Chem. 2000, 65,8041 .

[46] Fukuhara, G.; Mori, T.; Wada, T.; Inoue, Y. J. Org. Chem. 2006, 71,8233 .

[47] Lu, R.; Yang, C.; Cao, Y.; Wang, Z.; Wada, T.; Jiao, W.; Mori, T.; Inoue, Y. Chem. Commun. 2008, 2008, 374.

[48] Fukuhara, G.; Mori, T.; Wada, T.; Inoue, Y. Chem. Commun. 2005, 4199.

[49] Lu, R.; Yang, C.; Cao, Y.; Wang, Z.; Wada, T.; Jiao, W.; Mori, T.; Inoue, Y. J. Org. Chem. 2008, 7695.

[50] Gao, Y.; Wada, T.; Yang, K.; Kim, K.; Inoue, Y. Chirality 2005, $17, \mathrm{~S} 19$.

[51] Gao, Y.; Inoue, M.; Wada, T.; Inoue, Y. J. Inclusion Phenom. Macrocyclic Chem. 2004, 50, 111.

[52] ]Inoue, Y.; Yamasaki, N.; Yokoyama, T.; Tai, A. J. Org. Chem. 1993, 58, 1011.

[53] Inoue, Y.; Ikeda, H.; Kaneda, M.; Sumimura, T.; Everitt, S. R. L.; Wada, T. J. Am. Chem. Soc. 2000, 122, 406.

[54] Inoue, Y. Sentan Kagaku Shirizu 2003, 1, 77.

[55] Kaneda, M.; Nishiyama, Y.; Asaoka, S.; Mori, T.; Wada, T.; Inoue, Y. Org. Biomol. Chem. 2004, 2, 1295.

[56] Inoue, Y. Kagaku Kogyo [Tokyo, Jap.] 2006, 59, 152.

[57] Inoue, Y.; Tsuneishi, H.; Hakushi, T.; Tai, A. J. Am. Chem. Soc. 1997, 119, 472.

[58] Yang, C.; Mori, T.; Wada, T.; Inoue, Y. New J. Chem. 2007, 31, 697.

[59] Wang, Q.; Yang, C.; Ke, C.; Fukuhara, G.; Mori, T.; Liu, Y.; Inoue, Y. Chem. Commun. 2011, 47, 6849. 